\title{
Scientists race to develop a blood test for vCJD
}

As the bovine spongiform encephalopathy (BSE) crisis continues to intensify in Europe, the United States has so far managed to keep cattle infected with the disease out of the country. However, the US authorities are concerned that the human form of BSE, variant Creutzfeldt-Jakob disease (vCJD), might enter its shores through people who lived in Europe at the height of the BSE epidemic.

The US has banned blood donations from people in this category (Nature Med. 5,$7 ; 1999$ ) but there remains the remote chance that surgical procedures on infected people could spread vCJD, or that imported veterinary pharmaceuticals or food additives may be contaminated with BSE. Hence research into a preclinical test for the presence of the mutated prion is intensifying.

Robert Gallo, codiscoverer of HIV and director of the Institute of Human Virology in Baltimore, has become involved in research to develop a cheap and reliable blood test for vCJD. $\mathrm{He}$ has taken on the role of the principle invest gator and head of the scientific advisory board of Prion Developmental Laboratories (PDL), a private Marylandbased company. PDL represents a threepart research collaboration between Case Western Reserve University, the University of Maryland Biotechnology Institute and the Institute for Basic Research and Developmental Disabilities in New York.

Current efforts to develop a blood test for prion diseases center on methods of enriching the pathogenic prion protein. One possible route is the use of plasminogen, which sticks to rogue but not normal prion protein (Nature, 408, 479; 2000). "If there are any [abnormal] prions in vCJD blood, plasminogen should be able to pick them out," says Adriano Aguzzi of the University Hospital, Zurich. Aguzzi's group has licensed this technology to commercial organizations.

Another approach may come from the Canadian-based company, Caprion Pharmaceuticals, which claims to have developed "antibodies selective for the infectious form of the prion protein," says Clarissa Desjardins, vice president for business development. "The epitope that we are concentrating our efforts on is highly conserved in all species," she adds. The antibodies have been licensed

to IDEXX laboratories in Maine-the largest veterinary laboratory in the USwhich hopes to develop them for the detection of BSE, scrapie and related livestock diseases.

Despite these efforts, Paul Brown, chair of the US Transmissible Spongiform Encephalopathies (TSE) Advisory Committee and a prion expert at the National Institute of Neurological Disorders and Stroke, reminds us that "the question of infectiv-

ity in the blood of humans incubating vCJD remains open." No firm evidence exists for the presence of the prion in blood or for infection via the blood route. Most attempts to isolate the infectious agent from the blood of experimentally or naturally infected ruminants have failed, as have attempts to detect the vCJD agent in humans. And even if vCJD is detected in blood, this finding may not translate into a risk of infection.

\section{More sensitive tests for BSE needed}

\section{As concern about bovine spongiform en-} cephalopathy (BSE) increases in the US, it is perhaps surprising to learn that the US Food and Drug Administration has not yet approved a test to identify infected cattle (Nature, 409, 649; 2001) although several such post-mortem assays are available in Europe.

The standard experimental method used to test for infectivity by transmissible spongiform cephalopathies (TSE) involves inoculating a sample of infected material (neuronal tissue) into the brain of a laboratory mouse and then monitoring the animal. Such tests can take up to 12 months to yield a result.

Assays for the rapid de-

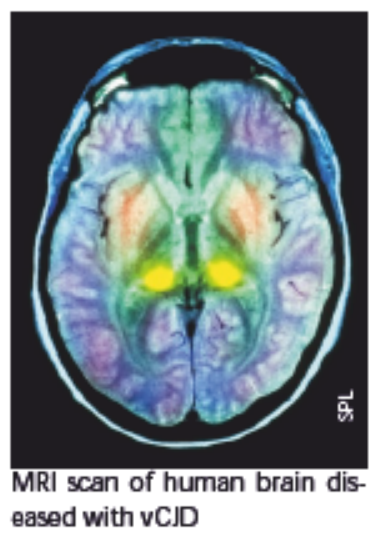

tested using one of these approved tests before the animals can be sold as beef. Testing became mandatory on 1 January for all "at risk" animals and as of 1 July, all cows aged over $\mathbf{3 0}$ months have to be tested-based on fact that the incubation period for BSE ranges from 4-6 years.

The Blo-Rad test is presently the most sensitive (Nature, 409, 476; 2001). "It is ten times more sensitive than the Irish test and thirty times more sensitive than the Swiss test," says Jean-Phillipe Desyls of the Prion research group at the CEA. However, there have been several reports from veterinary authorities across the EC that the test throws up hundreds of false positives. Desyls attributes this to technicians not havtection of prion protein by western blotting or ELISA techniques are quicker and more economical than this infectivity bioassay, but are not as sensitive, as it is not clear whether they can identify animals incubating the disease. All such tests involve the digestion of prion protein in infected tissue with a proteinase. Based on the fact that pathogenic prion protein is resistant to proteinase treatment, the abnormal prion material can be identified using antibodies.

To date, the European Commission (EC) has approved three such tests: the Bio-Rad test developed by the Commisariat a l'Energie Atomique (CEA) in France; Prionics-Check developed by Prionics AG in Switzerland; and the Enfer test system developed by Enfer Technology in Ireland.

New regulations in Europe require that brain tissue from slaughtered cattle be ing been trained properly on how to do the test. Although it uses a simple ELISA technique, "you have to have done it before to know how to read a positive result," he says.

Five more tests, focused on the ability to distinguish between different TSEs, are currently under examination by the EC. And there is also hope that the increased research into this area will yield a test and better still, a cure. On page 361 of this issue, Miele et al., report that the EDRF protein may act as a diagnostic marker for infection in cattle since it is expressed at lower levels in the blood of mice and sheep infected with TSE. It is not yet known whether EDRF is also downregulated in the blood of humans with vCJD and, if so, at what stage in the infection process.

Laura Bonetta, Bethesda 